\title{
COMMENTARY
}

\section{Sex Addiction: the Chicken-and-Egg Dilemma of Diagnosis}

\author{
Michael D. Berry*
}

In May 2013, the American Psychiatric Association will release the next version of the Diagnostic and Statistical Manual of Mental Disorders (DSM-V). Interestingly, sex addiction, despite significant attention from mainstream media, will be omitted from the manual. This omission presents a challenge to clinicians who treat sex addiction, and researchers aiming to further our understanding of the issue. This commentary outlines some of the reasons sex addiction was not included in the DSM-V, including a 'chicken-and-egg' conundrum, which makes it difficult to generate research without a clear diagnosis, and difficult to establish a definitive diagnosis without a supportive body of research.

The American Psychiatric Association (APA) has spoken. Sex addiction will not-in any way, shape or form-appear in DSM-V, the forthcoming iteration of the Diagnostic and Statistical Manual of Mental Disorders (American Psychiatric Association, 2012). Rather than settling the issue, however, this exclusion is likely to intensify the debate about whether sex addiction is a meaningful and valid clinical category. There is, after all, big money in the treatment of such a titillating psychosocial concern. Yet, the diffuse, and often contradictory, clinical conceptualizations of problematically excessive sexual behavior are a bane to those who hope to develop clear, broadly applicable treatment protocols. Additionally, the absence of a consensus definition is a logistical problem for researchers who aim to secure funding and support for projects examining the manifestation or treatment of hypersexuality. Without an accepted diagnosis, what exactly are we proposing to study, and to treat?

\footnotetext{
* University College London, United Kingdom michael.berry.10@ucl.ac.uk
}

In general, researchers agree that we have a very incomplete understanding of the causes of hypersexuality (Kaplan and Krueger 2010). In fact, we don't have a unified conceptualization of what sex addiction is (Kingston and Firestone 2008), or whether it is even a real condition (Moser 2011). This is, in large measure, due to a paucity of highquality empirical research. Interestingly, this relative scarcity, and the absence of a unified theory of causation, presents a 'chickenand-egg' dilemma. Without an accepted diagnostic category, it is difficult to clearly formulate (much less secure funding for) research that will further our understanding of an evidently real problem; however, in the absence of research that establishes a clear clinical picture of sexual addiction, it is difficult to justify any proposed diagnosis. Without empirical evidence, we can't agree on a diagnosis; without a diagnosis, we can't get empirical evidence.

Many practitioners, however, are undeterred by the lack of an accepted diagnostic framework; and, to the good fortune of sex addicts (or 'sex addicts,' if you prefer), many of these clinicians are highly trained, highly 
skilled professionals. Yet, while it can hardly be said that charlatanism is rampant in the field of sex addiction, it is clear that opportunism is a problem in this treatment area. The popular media's sensationalizing of celebrity 'sex addicts' and dubiously credible reports of epidemic sexual compulsion serve as convenient marketing tools for profitdriven 'therapists' with varyingly credible credentials. Moreover, celebrities routinely offer tearful confessions of 'sexual addiction' as a kind of public excuse for their transgressions, further publicizing, and perhaps legitimating, the issue in the public's view. From sports stars like Tiger Woods to film stars like Russell Brand and government figures like Lord Laidlaw, role models have been thrust into the limelight of celebrity sex addiction. Role models who happen to be-or who have at least been claimed to be-sex addicts. A cynical observer might speculate that for both celebrities and non-celebrities, 'sex addiction' is often used as an excuse when one's sexual indiscretions have been exposed. And indeed researchers have suggested that the diagnosis is applied in a wide range of cases, from discovered infidelity to unconventional sexual tastes, to simple dissonance between partners' respective views of sexuality (Levine 2010).

The hazy diagnostic picture makes epidemiological estimates quite difficult. Researchers in the U.S. have suggested an overall prevalence of 3-6 per cent amongst American adults, although these figures are rather dated, and it is unclear exactly how they were determined in the first place (Black 2000, Coleman 1992). Data-even speculative data-is even scarcer for the UK. We do know that men are much more commonly 'diagnosed' as sex addicts, though we don't know why. This is one of the clear problems in such a research-scarce area.

In addition to the shortage of research data to clarify the epidemiological picture, or validate a particular diagnostic framework (and, indeed, there have been many varyingly divergent labels suggested), pragmatic concerns have been voiced. For instance, if a sexual addiction category were included in the DSM, might it be used as a legal defense for sexual crimes, like exhibitionism, or sexual assault? Interestingly, the latter concern did not deter the APA from including 'pedophilic disorder' in DSM-V. While the same legal concerns apply for both categories, a larger body of interdisciplinary research justifies the inclusion of pedophilic disorder. Additionally, some aspects of pedophilic behavior-such as contact with vulnerable underage populations, and the use of child pornography-are more clearly entrenched in legal statute than many of the behaviors associated with sex addiction-such as use of legal pornography, marital infidelity, and hazier legal areas such as prostitution. Thus, the socio-political field further confounds researchers and clinicians already frustrated by a shortage of research on sex addiction.

In my assessment, at least, it is quite clear that we need significantly more research on sex addiction. Despite the skeptical, or antidiagnostic, stance of some researchers (Moser 2011, Winters 2010), the primary obstacles are logistic. Ours is an era where funding is increasingly limited, and a substantial portion is allocated, understandably, to more quantifiably drastic health concerns and 'proven earners' where clear, tangible outcomes can be expected. Unsurprisingly, it's very difficult to secure funds for sex addiction research. Additionally, like other sexual health concerns, there is shame associated with sexual addiction. If sex addiction is in some measure symptomatic of a maladaptive response to difficult thoughts and feelingsand there is good reason to think this is part of the clinical picture (Kafka 2010)-then sex addicts could be less equipped to manage and communicate their experience, and less enthusiastic to participate in research than individuals with more ideologically neutral health concerns. And, of course, the crux of my argument here is that the absence of an accepted conceptual framework is a key factor. Researchers from all areas are likely familiar with the challenge of securing funding for ill defined, or significantly contested, 
research topics. It is very difficult to get money to study sex addiction without being able to say definitively what it is.

So, what can we do? Part of the solution likely consists in the development of highquality, but small-scale research projects. As with anything, in the health sciences it is usually easier to mobilize smaller resource pools, and secure more modest research grants. Smaller projects can also be more feasibly hosted or sponsored by independent institutions and private clinics, and indeed there are a number of researchers and practitioner-investigators conducting modestly sized but important research projects. Obviously, smaller projects tend to be less scientifically convincing than the big ones. But it's a commendable, and necessary, start.

Another possibility is that drug companies, with their abundant research and development dollars, may start funding research on treatment avenues. In fact, some creative physicians already prescribe antidepressants in the SSRI family to treat sex addicts. It has long been known that SSRIs incidentally affect sex drive and sexual responsiveness (for the latter reason, they are also regularly used to treat premature ejaculation). It is not unreasonable to think that similar compounds, or even the same drugs, could be approved for the treatment of sexual addiction. For the time being, though, without an officially diagnosable pathology to treat, national health agencies are unlikely to recertify off-label drug therapies for such a purpose.

Even if a drug company were to introduce a clinically efficacious treatment, this is ultimately only a partial solution. In addition to the inevitable non-responders and ineligible patients, drug therapies only address one facet of a multidimensional problem. Despite the availability of some very thoughtful speculation and promising preliminary work, we really don't know if sex addiction has an exclusively, or even primarily, physiological pathway. Especially since the advent of Viagra, many sex therapists have loudly decried the exclusive use of drug therapies, on the basis that they fail to address the inevitable psychological and social dimensions of sexual problems. Sex addiction is subject to the same concerns.

Ultimately, the problems associated with sex addiction research are much bigger than just sex addiction. The sexual health disciplines are fractured, largely by sub-disciplinarily, and by the endemic competition for resources, especially research dollars, that accompanies contemporary health sciences research. Psychotherapists and psychological researchers often take a different tack from bio-medical researchers, and clinicians often work in environments where interdisciplinary treatment is limited to referral, rather than direct collaboration. It just so happens that these clinical and research obstacles are particularly challenging for a diagnostic issue as inchoate as sex addiction.

\section{References}

American Psychiatric Association 2012 American Psychiatric Association Board of Trustees Approves DSM 5. APA News Release No. 12-43, January 12, 2012. Available at http://www.dsm5.org/newsroom/pages/pressreleases.aspx [Last accessed 10 December 2012].

Black, D W 2000 The Epidemiology and Phenomenology of Compulsive Sexual Behavior. CNS Spectrums, 5: 26-35.

Coleman, E 1992 Is Your Patient Suffering from Compulsive Sexual Behavior? Psychiatric Annals, 22: 320-325.

Kafka, M P 2010 Hypersexual Disorder: A Proposed Diagnosis for DSM-V. Archives of Sexual Behavior, 39: 377-400.

Kaplan, M S and Krueger, R B 2010 Diagnosis, Assessment, and Treatment of Hypersexuality. Journal of Sex Research, 47: 181-198.

Kingston, D A and Firestone, P 2008 Problematic Hypersexuality: A Review of Conceptualization and Diagnosis. Sexual Addiction and Compulsivity, 15: 284-310.

Levine, S B 2010 What Is Sexual Addiction. Journal of Sex \& Marital Therapy, 36: 261-275. 
Moser, C 2011 Hypersexual Disorder: Just More Muddled Thinking. Archives of Sexual Behavior, 40: 227-229.
Winters, J 2010 Hypersexual Disorder: A More Cautious Approach. Archives of Sexual Behavior, 39: 594-596.

How to cite this article: Berry, M 2013 Sex Addiction: the Chicken-and-Egg Dilemma of Diagnosis. Opticon1826, (15): 8, pp. 1-4, DOl: http://dx.doi.org/10.5334/opt.bf

Published: 4 July 2013

Copyright: (c) 2013 The Author(s). This is an open-access article distributed under the terms of the Creative Commons Attribution 3.0 Unported License (CC-BY 3.0), which permits unrestricted use, distribution, and reproduction in any medium, provided the original author and source are credited. See http://creativecommons.org/licenses/by/3.0/.

]u[ Opticon1826 is a peer-reviewed open access journal published by

]u[ Ubiquity Press 